\title{
Vision loss in granulomatosis with polyangiitis: when prednisone is the problem, not the solution
}

\author{
Laura Donaldson $^{1} \cdot$ Christian Pagnoux ${ }^{2} \cdot$ Edward Margolin $^{1,3}$ (])
}

Received: 13 August 2021 / Revised: 16 September 2021 / Accepted: 17 September 2021 / Published online: 26 September 2021

(c) International League of Associations for Rheumatology (ILAR) 2021

\begin{abstract}
A 38-year-old man presented with diffusely red left eye (LE). Visual acuity was $20 / 20$ bilaterally; there was severe conjunctival injection and mild engorgement of episcleral vessels. A diagnosis of simple episcleritis was made; however, swelling of soft tissues of the right ear was noticed; thus, systemic inflammatory work-up was initiated. Erythrocyte sedimentation rate (ESR) and C-reactive protein (CRP) were both elevated; rheumatoid factor and proteinase 3 antineutrophil cytoplasmic antibody (PR3-ANCA) titers were positive. Creatinine level was $5 \mathrm{mg} / \mathrm{dL}$; thus, renal biopsy was performed demonstrating necrotizing crescentic glomerulonephritis. Diagnosis of granulomatosis with polyangiitis (GPA) was made, and treatment with intravenous methylprednisolone followed by plasma exchange and rituximab commenced. He was discharged on $75 \mathrm{mg}$ of oral prednisone.

Seven months later, while on $7.5 \mathrm{mg}$ of prednisone and rituximab, he noticed blurry vision in the right eye (RE). Vision was 20/40 in RE and 20/20 in LE. Ophthalmoscopy showed dome-shaped elevation of the right macula. Optical coherence tomography confirmed
\end{abstract}

large serous retinal detachment (Fig. 1A). Diagnosed of central serous chorioretinopathy (CSCR) secondary to prednisone use was made. Prednisone was stopped, and 1 month later, subretinal fluid almost completely resolved (Fig. 1B) with subjective improvement in vision. His GPA remained well-controlled on maintenance rituximab, with creatinine stable at $1.66 \mathrm{mg} / \mathrm{dL}$ and no ocular inflammation.

CSCR is an ocular condition in which serous fluid accumulates under central retina producing decreased vision and metamorphopsia (distortion and curving of linear objects). It is associated with glucocorticoid use, and usually resolves after steroids are discontinued. This case illustrates that visual loss is not always due to active ocular disease, and in this case, increasing prednisone dose would have exacerbated the underlying condition. Glucocorticoids are commonly used in the treatment of rheumatological disorders; thus, rheumatologists should be aware of CSCR.

Edward Margolin

Edward.Margolin@sinaihealthsystem.ca

1 Department of Ophthalmology and Vision Sciences, University of Toronto, Toronto, Ontario, Canada

2 Division of Rheumatology, Department of Medicine, University of Toronto, Toronto, Ontario, Canada

3 Division of Neurology, Department of Medicine, University of Toronto, 801 Eglinton Ave West, Suite 301, Toronto, ON M5N 1E3, Canada 
Fig. 1 A Macular ocular coherence tomography (OCT) demonstrating dramatic serous retinal detachment affecting right macula (left panel), while left macula (right panel) is nor$\mathrm{mal} /$ flat. B OCT of the macula 1 month later demonstrating almost complete resolution of subretinal fluid previously seen in the right macula
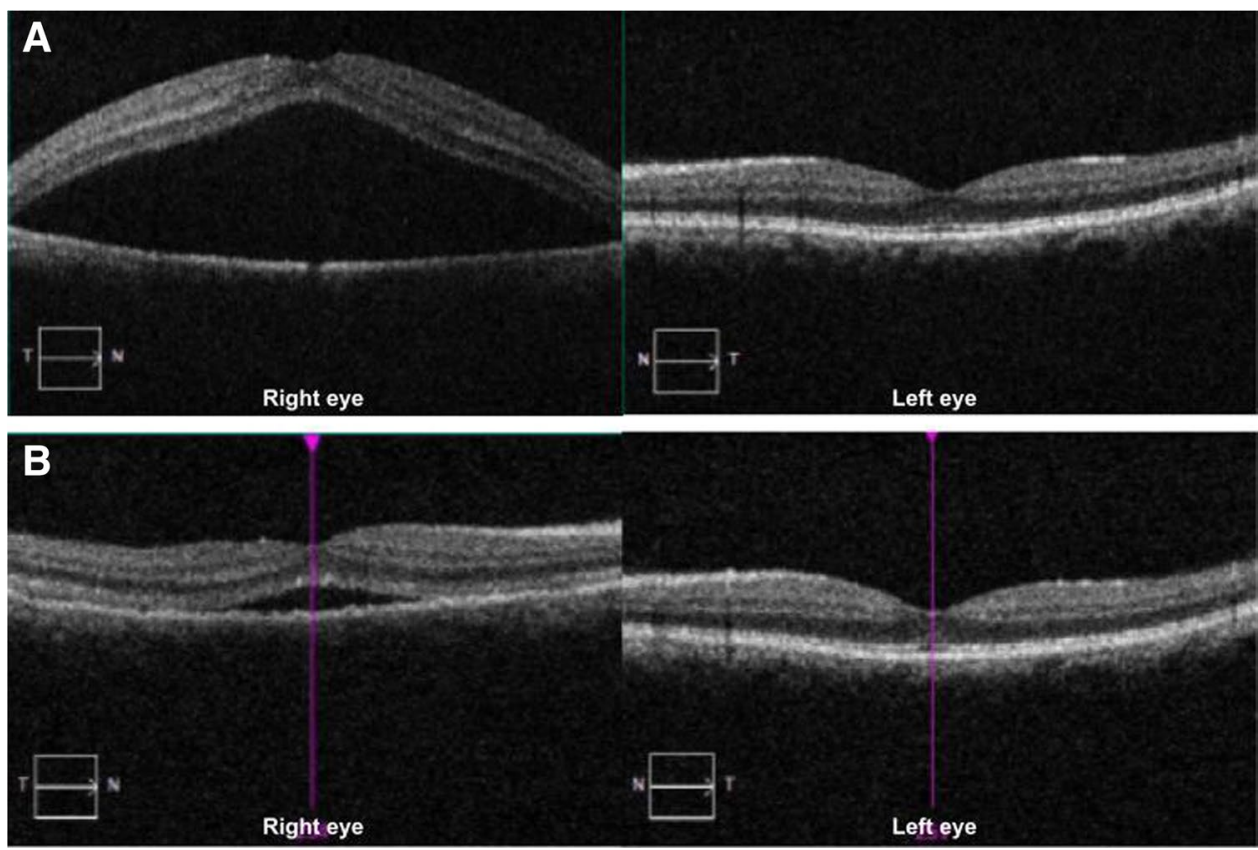

\section{B}

Publisher's note Springer Nature remains neutral with regard to jurisdictional claims in published maps and institutional affiliations.

\section{Declarations}

Ethics review was not required, and patient consent was uploaded separately.

\section{Disclosures None.}

